\title{
The effect of desmethylimipramine on the absorption of alcohol and paracetamol
}

\author{
R. C. HALL * \\ M.B., Ch.B., M.Sc. \\ R. CARTER \\ M.B., Ch.B. \\ DEIRDRE BROWN \\ M. J. KenDALL \\ M.D., M.R.C.P.
}

Department of Therapeutics and Clinical Pharmacology, Medical School, Birmingham B15 2TJ

\begin{abstract}
Summary
Many drugs can delay gastric emptying and may thereby impair the absorption of other drugs. Desmethylimipramine (DMI) is an antidepressant with anticholinergic properties which might be expected to delay the rate of gastric emptying. In five out of six subjects, DMI significantly delayed both the absorption of alcohol (assessed by a breathalyser) and of paracetamol, and there was a correlation between the two. The breathalyser can therefore be used to predict the extent to which one may impair the absorption of another drug by its effect on gastrointestinal motility.
\end{abstract}

\section{Introduction}

The rate of gastric emptying is an important factor in determining the rate of absorption of drugs since uptake from the stomach is slow whereas absorption from the upper intestine is usually rapid (Prescott, 1974). The rate of emptying varies from person to person and can be changed by food, posture and disease. It can also be altered by a number of different drugs particularly those which modify the actions of the autonomic nervous system (Nimmo et al., 1973; Manninen et al., 1973; Prescott, 1974). This means that patients receiving more than one drug may fail to respond because one substance is impairing the absorption of the other. Since there are many drugs which could disturb the normal motility of the gut, the problem is to determine which preparations will increase or decrease the rate of gastric emptying to an extent which could be clinically important.

Desmethylimipramine (DMI) is an antidepressant which has some anticholinergic properties and causes dry mouth, urinary retention, constipation and drowsiness (Klerman and Cole, 1965). It also inhibits gastric secretion in man (Baume and Powell, 1966) and studies in the rat have suggested that this is due to an action of the drug on the

\footnotetext{
* Present address: Department of Human Physiology and Pharmacology, University of Adelaide, South Australia.

Correspondence and reprint requests: $\mathrm{Dr}$ M. J. Kendall.
}

central nervous system (Pendleton et al., 1970; Ridley and Mann, 1973). Since DMI has these anticholinergic effects one might anticipate that it would delay gastric emptying and thereby impair the absorption of drugs from the gut. There is some evidence that it has this effect on phenylbutazone, the serum levels of which are reduced by administration of DMI (Consolo et al., 1970).

The work described in this paper was undertaken to determine whether DMI delays gastric emptying sufficiently to affect seriously the absorption of another drug and whether this can be predicted by examining its effect on the absorption of alcohol. Changes in the rate of absorption of alcohol can be used to detect changes in the rate of gastric emptying (Finch, Kendall and Mitchard, 1974). We have therefore compared the effects of DMI on alcohol absorption (using a breathalyser) with its effects on the absorption of paracetamol.

\section{Methods}

The studies were carried out on six healthy male volunteers. One was taking long-term phenobarbitone and phenytoin, but the others were not on any drugs. All participants fasted $12 \mathrm{hr}$ before each experiment and the same subjects were used throughout so that each subject acted as his own control.

\section{Alcohol absorption}

Each subject ingested $40 \mathrm{ml}$ absolute alcohol made up to $200 \mathrm{ml}$ with water and orange cordial. After ingestion, the mouth was rinsed several times with water. The blood alcohol levels were then determined by a Lions Mark II Intoximeter which measures the concentration of alcohol in the expired air. Measurements were made at 10-min intervals for up to $150 \mathrm{~min}$. The experiment was subsequently repeated following the ingestion of DMI $90 \mathrm{~min}$ before alcohol. Two doses of DMI were used: $75 \mathrm{mg}$ in six/six subjects and $25 \mathrm{mg}$ in five/six subjects. In a further study four subjects took the $25 \mathrm{mg}$ DMI dose $45 \mathrm{~min}$ before alcohol. 


\section{Paracetamol}

All subjects ingested $40 \mathrm{mg} / \mathrm{kg}$ paracetamol. A cannula was inserted in an antecubital vein and blood samples $(10 \mathrm{ml})$ were taken for up to $6 \mathrm{hr}$ after ingestion of the drug at $10,15,30,45 \mathrm{~min}, 1 \cdot 0,1 \cdot 5$, $2 \cdot 0,2 \cdot 5,3 \cdot 0,3 \cdot 5,4 \cdot 0,5 \cdot 0$ and $6 \cdot 0 \mathrm{hr}$. Between samples, the cannula was perfused slowly with $0.15 \mathrm{~mol} / \mathrm{l}$ saline, so that about $700-800 \mathrm{ml}$ was given over the course of the $6 \mathrm{hr}$. Blood samples were centrifuged and the plasma was taken off and stored at $-20^{\circ} \mathrm{C}$. Analyses were carried out within 1 week of sampling. The study was subsequently repeated following the ingestion of DMI $90 \mathrm{~min}$ before the paracetamol. The procedure was the same as that used in the alcohol absorption studies.

\section{Estimation of paracetamol}

The plasma was extracted by the method of Berry and Grove (1973). The extracted residue was then dissolved in redistilled ethanol and the sample read at a wavelength of $250 \mathrm{~nm}$ on an SP 500 u.v. Spectrophotometer (J. Grove, personal communication). Standard curves were prepared in plasma. In our hands, the reproducibility of the method in the concentrations measured was $\pm 2 \%$.

\section{Drugs and reagents}

Desmethylimipramine (Pertofran, Geigy; $25 \mathrm{mg}$ tablets); paracetamol (Panadol, Winthrop; $500 \mathrm{mg}$ tablets); ethanol (redistilled); ammonium sulphate (Analar grade); ether (redistilled).

\section{Results}

The effect of $75 \mathrm{mg}$ DMI on the absorption of alcohol was the same as its effect on paracetamol absorption in each case. However, in five subjects, $\frac{3}{8}$ the absorption was impaired whilst in one person the $\propto$ administration of DMI enhanced the absorption of $c$ those substances. The results for this person, who $\overrightarrow{\vec{F}}$ was clinically well and not on any medication, have therefore been considered separately.

\section{Alcohol}

The effects of DMI on the absorption of alcohol in five of the six subjects are shown in Fig. 1. In the absence of DMI, the mean peak concentration was $73.4 \pm 7.1 \mathrm{mg} / 100 \mathrm{ml}$ and this occurred $33.6 \pm 3.0$ min after ingestion of alcohol. Pre-treatment with $75 \mathrm{mg}$ DMI $90 \mathrm{~min}$ before alcohol caused a signifi- 8 cant reduction in the peak concentration to $47 \cdot 6 \pm 1 \cdot 43$. $(P<0.05)$ and a delay in the time of the peak to in $53.6 \pm 8.0 \mathrm{~min}$. The blood alcohol levels in subjects pre-treated with $75 \mathrm{mg}$ DMI were significantly lower of than in controls from $30-50 \mathrm{~min}(P<0.02)$ and also $60 \mathrm{~min}(P<0.05)$ after ingestion. Thereafter, there was no significant difference between control and treated subjects. In four subjects the lower dose of $\rightarrow$ $25 \mathrm{mg}$ DMI taken $90 \mathrm{~min}$ before alcohol had an effect on alcohol absorption intermediate between control and $75 \mathrm{mg}$ DMI. Three of these subjects also took $25 \mathrm{mg}$ DMI $45 \mathrm{~min}$ before alcohol an there was no significant difference between the results and those obtained when $25 \mathrm{mg}$ DMI waş given 90 min before ingestion. In the sixth subject, DMI 25 and $75 \mathrm{mg}$ taken $90 \mathrm{~min}$ before the alcohol increased the peak concentration of alcohol to 73.0 and $87.0 \mathrm{mg} / 100 \mathrm{ml}$ respectively from a control peak $50.4 \mathrm{mg} / 100 \mathrm{ml}$.

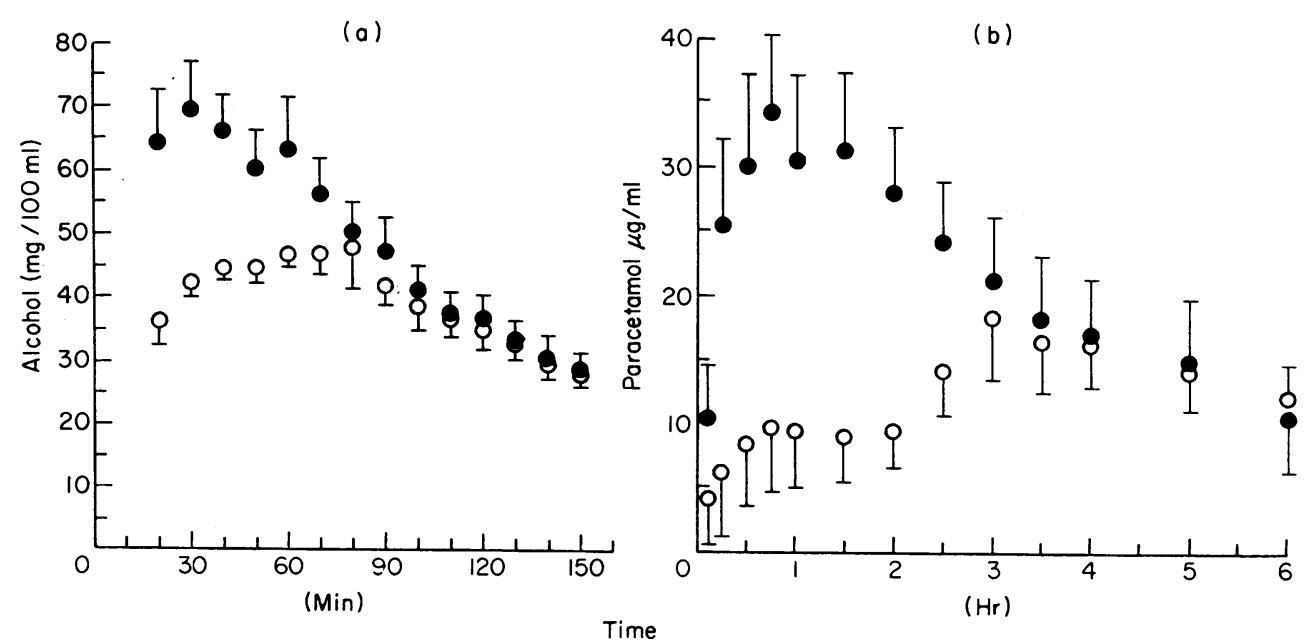

FIG. 1. Effect of desmethylimipramine (DMI) on the absorption of (a) alcohol and (b) paracetamol. Both figures show the means ( \pm 1 s.e. mean) of five subjects before $(O)$ and after $(O)$ DMI administration. 


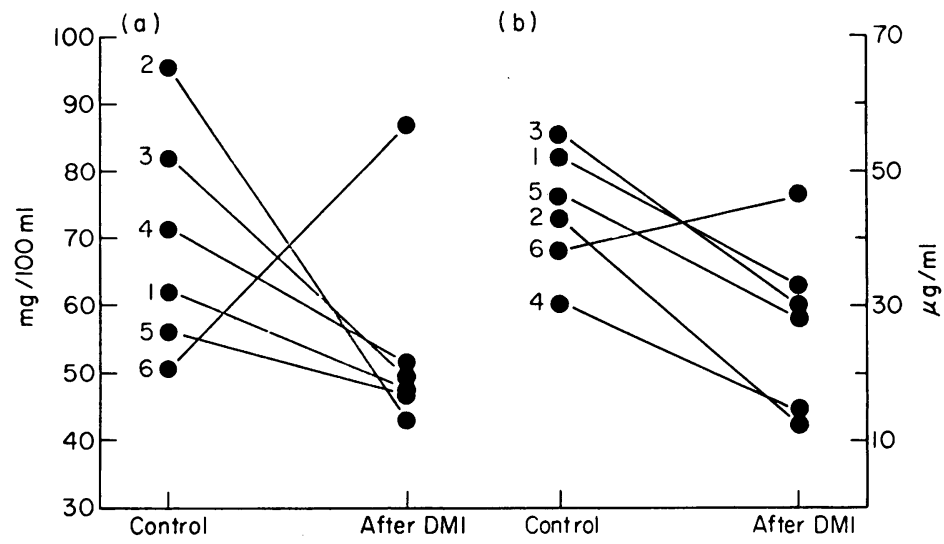

FIG. 2. The effect in individual subjects of $75 \mathrm{mg}$ DMI given $90 \mathrm{~min}$ before the study on peak concentrations of (a) alcohol and (b) paracetamol.

\section{Paracetamol}

DMI had qualitatively similar effects on the absorption of paracetamol to those observed with alcohol. Fig. 1 shows the mean ( \pm s.e. mean) paracetamol levels before and after DMI in five individuals. DMI, $75 \mathrm{mg}$, significantly depressed the peak concentration of paracetamol from a control of $33.6 \pm 3.0 \mu \mathrm{g} / \mathrm{ml}$ to $23.8 \pm 4.3(P<0.01)$ and also significantly delayed the time of the peak from $50.8 \pm 10.8 \mathrm{~min}$ in controls to $187 \pm 45.6 \mathrm{~min}$ $(P<0.05)$ in treated subjects.

The mean plasma concentrations of paracetamol were significantly lower in DMI-treated subjects at 30 and $150 \mathrm{~min}(P<0.05)$ and at 60 and $120 \mathrm{~min}$ $(P<0.02)$. No significant difference was detectable thereafter up to $6 \mathrm{hr}$. In three subjects, $25 \mathrm{mg}$ DMI 90 min before paracetamol had an intermediate effect between control and the higher dose of DMI. The effect of DMI on paracetamol absorption in the sixth was comparable with its effect on alcohol absorption, in both doses it caused an increase in the peak concentration of paracetamol.

The peak concentrations of paracetamol and alcohol before and after $75 \mathrm{mg}$ DMI are shown for individual subjects in Fig. 2. This shows not only the anomalous behaviour of subject 6 , but also demonstrates that the other results are fairly comparable. Thus, DMI markedly reduced the peaks of subject 2 but had a much smaller effect in subject 5 in both the alcohol and the paracetamol study.

Figure 3 shows the correlation between peak plasma concentration of alcohol and paracetamol for all subjects and all treatments. There is a significant correlation between these peaks $(r=: 0.66$; $P<0.01$ ).

\section{Discussion}

These results show that DMI reduces the peak

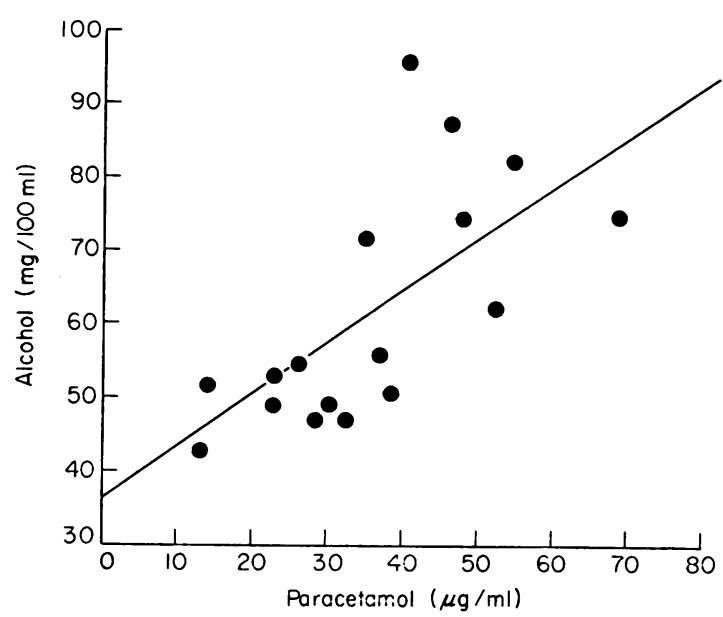

Fig. 3. Correlation between peak alcohol concentrations and peak paracetamol concentrations in all subjects.

concentration and delays the absorption of paracetamol. Similar effects were observed on the absorption of alcohol and, furthermore, there was a significant correlation between changes in alcohol and paracetamol concentrations providing further evidence that the breathalyser is a useful simple technique for monitoring changes in the absorption of drugs from the gastrointestinal tract (Finch, Kendall and Mitchard, 1974). In this respect it is especially noteworthy that in the one subject in whom DMI had the unexpected effect of increasing the peak alcohol concentration a similar effect was observed on the absorption of paracetamol.

However, while changes in alcohol concentration were related to changes in paracetamol concentration, DMI had a very much more marked effect on the rate of absorption of paracetamol, for whilst the 
mean peak alcohol concentration was delayed by 20 min, the paracetamol peak occurred approximately 120 min later. This difference between the rate of absorption of the two drugs may be a reflection of the fact that alcohol is absorbed from the stomach, albeit slowly, while paracetamol is only absorbed to any extent from the small intestine. Because of the limited absorption of alcohol from the stomach the breathalyser may underestimate a decreased rate of absorption of drugs.

While the present study cannot fully explain the way in which DMI exerts its effect on the absorption of drugs, it is consistent with the anticholinergic properties of the drug. Reducing the dose of DMI reduced but did not abolish the effect of the drug and, furthermore, in four subjects, changing the time of administration from $90 \mathrm{~min}$ to $45 \mathrm{~min}$ before alcohol did not alter its effect on the absorption of alcohol. The observation that DMI had the opposite effect in one subject cannot be explained from the present study but emphasizes the dangers of making even qualitative generalizations on drug metabolism.

The administration of DMI not only delays the peak but also reduces the maximum concentration attained. Clinically this may be important since it implies that anticholinergic agents could prevent other drugs from ever reaching a therapeutic level in the blood. In the case of alcohol, there is some evidence that the total amount absorbed may be reduced (Finch et al., 1974) possibly because more is metabolized in the gut. However, there is insufficient evidence to make any such comment about paracetamol and we feel that the lower peak results from the slower rate of absorption leading to a flatter type of drug level/time profile. Presumably DMI not only delays the rate of gastric emptying but also reduces upper intestinal motility.

Further studies of this sort are needed since it is important to assess the effect of DMI and other preparations with anticholinergic properties on the absorption of drugs such as digoxin, anticonvulsants气 and antibiotics, since fluctuation in the plasma con- 3 centration of these substances may have serious $\varrho$ clinical consequences.

\section{Acknowledgments}

We are grateful to Dr A. Clayton of the Transportation $\overline{\bar{\sigma}}$ Department in the University of Birmingham for the loan of the intoximeter and to Ruth Gibson for her help in carrying out the studies.

M.J.K. is a MRC Clinical Research Fellow.

\section{References}

BAUME, P. \& Powell, K.C. (1966) Inhibition of human basal gastric secretion by desmethylimipramine. Medical Journa of Australia, 2, 596.

BERRY, D.J. \& GROVE, J. (1973) Emergency toxicological screening for drugs commonly taken in overdose. JournalN of Chromatography, 80, 205.

Consolo, S., Morselli, P.L., Zaccala, M. \& Garattini, S.O (1970) Delayed absorption of phenylbutazone caused by desmethylimipramine in humans. European Journal of Pharmacology, 10, 239.

Finch, J.E., Kendall, M.J. \& MitchaRd, M. (1974) An을 assessment of gastric emptying by breathalyser. BritishJournal of Clinical Pharmacology, 1, 233.

KLERMAN, G.L. \& Cole, J.D. (1965) Clinical pharmacology of imipramine and related antidepressant compounds. $\frac{\hat{S}}{\supset}$ Pharmacological Reviews, 17, 101.

Manninen, V., Apajalahti, A., Melin, J. \& Karesoja, A. $\vec{\varphi}$ (1973) Altered absorption of digoxin in patients gi propantheline and metoclopromide. Lancet. i, 398.

Nimmo, J., Heading, R.C., Tothill, P. \& Prescott, L (1973) Pharmacological modification of gastric emptying: effects of propantheline and metoclopromide on para- $\bar{\partial}$ cetamol absorption. British Medical Journal, 1, 587.

Pendleton, R.G., Bartakovits, P., Miller, D.A., Mann, W.A. \& RIDLEY, P.T. (1970) Studies indicating a central antisecretory site of action for desmethylimipramine. $\overrightarrow{\vec{D}}$ Journal of Pharmacology and Experimental Therapeutics, $\frac{0}{3}$ 174, 421.

PrescotT, L.F. (1974) Gastric emptying and drug absorption. British Journal of Clinical Pharmacology. 1, 189.

RIDLEY, P.T. \& MANN, W.A. (1973) Central antisecretory action of desmethylimipramine in the rat. American 3 Journal of Digestive Diseases 18, 493. 